\title{
1798-1976. Le français référentiaire des élites
} égyptiennes

Amr Helmy Ibrahim

\section{(2) OpenEdition \\ Journals}

Édition électronique

URL : https://journals.openedition.org/dhfles/148

DOI : $10.4000 /$ dhfles. 148

ISSN : 2221-4038

Éditeur

Société Internationale pour l'Histoire du Français Langue Étrangère ou Seconde

Édition imprimée

Date de publication : 1 janvier 2007

Pagination : 131-147

ISSN : 0992-7654

Référence électronique

Amr Helmy Ibrahim, «1798-1976. Le français référentiaire des élites égyptiennes », Documents pour I'histoire du français langue étrangère ou seconde [En ligne], 38/39 | 2007, mis en ligne le 16 décembre 2010, consulté le 27 mai 2021. URL : http://journals.openedition.org/dhfles/148 ; DOI : https://doi.org/ $10.4000 /$ dhfles. 148

Ce document a été généré automatiquement le 27 mai 2021.

(c) SIHFLES 


\title{
1798-1976. Le français référentiaire des élites égyptiennes
}

\author{
Amr Helmy Ibrahim
}

\section{Qu'est-ce que l'élitisme?}

1 Le français a eu, dès le XVII ${ }^{e}$ siècle et pendant plus de deux siècles un double privilège : ce n'était pas la langue du peuple français mais d'une élite qui a su imposer ses usages au peuple au point de lui confisquer la légitimité de sa parole; du fait du rayonnement du savoir-faire, du savoir- penser et du savoir-être de cette élite, c'était une langue si légitimement véhiculaire qu'il n'y avait aucune honte à la considérer universelle.

Il y a là un tour de force dont Vaugelas a fixé très tôt le protocole :

Il y a sans doute deux sortes d'usages, un bon et un mauvais. Le mauvais se forme du plus grand nombre de personnes qui presque en toutes choses n'est pas le meilleur, et le bon au contraire est composé non pas de la pluralité mais de l'élite des voix [...] Voici donc comme on définit le bon usage. C'est la façon de parler de la plus saine partie de la cour conformément à la façon d'écrire de la plus saine partie des auteurs du temps. (Vaugelas 1981,10)

3 La langue devient ainsi un vecteur de modélisation des conduites et d'adhésion idéologique qui ne s'appuie ni sur la force brutale, ni sur le nombre ni, par conséquent, sur le terrorisme consensuel qui en découle.

4 La formulation de Vaugelas, plus de deux siècles avant l'œuvre de Pareto qui en fera le moteur de l'histoire et de l'économie, nous dit comment et surtout pourquoi l'élitisme construit, contre les pratiques et les usages du plus grand nombre, qu'il délégitime, un modèle de pratiques et un usage qui s'instituent en référence unique, universelle et exclusive de toute autre pratique ou usage.

De plus, le projet politique français à l'âge classique porte à sa maturité une tendance potentielle des langues : inscrire dans une nébuleuse de paradigmes prototypiques des significations qui se confondent avec les formes linguistiques dans lesquelles elles sont 
exprimées. Régir officiellement ces formes revient alors, sans avoir l'air d'y toucher, à régler au millimètre près rien moins que la production même du sens.

Pour parler comme Rivarol, dont le Discours sur l'universalité de la langue française (1783-1784) donne la mesure de la place que l'Europe des lumières réservait au français à l'époque de l'expédition d'Égypte, on peut dire qu'aucun peuple n'avait, depuis les Romains, bénéficié, comme les Français, d'une conjoncture aussi favorable à ce que sa langue devienne par sa pratique et l'imitation de ce qu'elle suggère, un aussi sûr vecteur d'excellence. Antoine de Rivarol, après avoir dressé la liste des bonnes raisons pour lesquelles tous les Européens se piquent de parler français et d'imiter les Français, n'écrit-il pas :

Nous sommes les seuls qui imitions les Anglais, et, quand nous sommes las de notre goût, nous y mêlons leurs caprices; nous faisons entrer une mode anglaise dans l'immense tourbillon des nôtres, et le monde l'adopte au sortir de nos mains. (Rivarol 1995, 161)

\section{Le paradoxe de Milner}

7 C'est en effet dans un pays occupé et dominé par les Anglais qu'émergera une élite qui aura choisi la référence française :

Tout ce que l'Égypte emprunta à l'Europe, dans l'ordre matériel aussi bien que dans l'ordre intellectuel, lui vint d'abord de la France. Si ses hautes classes furent quelque peu instruites, elles le furent par des maîtres français et dans les idées françaises ; le français devint même une langue officielle côte à côte avec l'arabe, et encore aujourd'hui, c'est en un français boiteux que les Anglais au service de l'Égypte échangent leurs lettres officielles. (Milner 1892 : 478)

On doit ce jugement, publié en 1892 dans L'Angleterre en Égypte, à Sir Alfred Milner, sous-secrétaire d'État au ministère égyptien des Finances pendant deux ans. La GrandeBretagne occupe l'Égypte depuis déjà dix ans. Lorsque les troupes britanniques évacueront définitivement l'Égypte au terme de 74 années d'occupation, l'empire sur lequel le soleil ne se couchait jamais n'aura pas pu éradiquer ni même réduire significativement la référence française implantée par Bonaparte en moins de trois ans (1798-1801) et relayée par une succession de choix élitaires que nous passerons en revue.

La semence française prend d'autant mieux qu'à l'arrivée de Bonaparte et pendant près d'un siècle l'Égypte n'a pas une aristocratie autochtone, tout au moins au sens européen de ce terme : une noblesse héréditaire et un ensemble suffisant de familles qui maintiennent dans leur descendance et leur clientèle immédiate les leviers du pouvoir, de la richesse et de la connaissance.

L'Égypte de l'époque a certes des groupes, des familles et des individus qui cultivent une forme ou une autre d'excellence intellectuelle ou se réclament d'une descendance qui leur impose des obligations sociales et des responsabilités politiques, mais ces îles ne donnent lieu à aucune conscience élitaire collective. L'on peut donc dire que même à cette échelle, c'est l'expédition de Bonaparte qui a réveillé en Égypte le fait élitaire, notamment dans sa fonction dynamique.

11 Il n'est que de voir ce Moustafa Kamel Pacha qui a 18 ans à la parution du livre de Milner et qui fera, trois ans plus tard, le 4 juillet 1895, à Toulouse, dans un français que lui envieraient bien des normaliens français de souche d'aujourd'hui, le premier grand 
discours nationaliste de l'Égypte moderne ${ }^{1}$. Il fondera, après avoir achevé ses études de droit en France, un journal ${ }^{2}$, l'un des premiers partis politiques dignes de ce nom ${ }^{3}$ ainsi que la première école comprenant un tiers d'élèves dispensés de frais de scolarité, un système dont s'inspireront les missions catholiques françaises en Égypte. Moustafa Kamel Pacha n'est pas l'exception mais le modèle, le prototype de cette nouvelle élite pour laquelle le français devient référentiaire.

\section{L'expédition savante de Bonaparte}

Bonaparte sait bien et il n'est pas seul en Europe à savoir que le ressort de la société égyptienne est cassé malgré les ingrédients de qualité dont elle a hérité. Il sait aussi, tout comme les Anglais, que l'Europe n'est pas viable sans la domination du monde et singulièrement de cet Orient dont l'Égypte, la Mésopotamie, l'Inde et la Chine constituent les passages obligés. Mais il pense aussi, ce dont doutent fort les Anglais, que c'est au cœur des vieilles civilisations que l'intelligence française, qui exprime la quintessence du dynamisme européen, doit rétablir, sous la houlette du plus éclairé des hommes, le nouvel Alexandre, l'équilibre du monde. C'est donc parmi les plus authentiquement croyants des lettrés musulmans, dans les familles de ces 3oulama' dont la raison d'être est la connaissance du monde que la sauce du projet foncièrement laïc du caporal Bonaparte prendra le mieux. Ils finiront par comprendre en profondeur, ces lettrés, que ce que les élites françaises ont acquis de haute lutte dans leur concurrence féroce avec les autres élites européennes et qu'elles étaient disposées - du fait d'une conjoncture exceptionnelle - à leur transmettre sans menacer le moins du monde les fondements idéologiques et culturels de leur foi, était cela même que leur civilisation de référence avait progressivement perdu, pour avoir, peut-être à cause, justement, de son éclatant équilibre, oublié que le monde ne se réduisait pas à elle et qu'aucun équilibre n'était définitivement acquis.

(a) aurait pu si ce n'était l'entrave anglaise - près d'un siècle d'actions ouvertement négatives à l'encontre de l'Égypte - et le passif ottoman - trois siècles de l'une des occupations les plus stériles que le pays ait connues en 4000 ans d'histoire - déboucher sur une renaissance qui aurait refait de l'Égypte un foyer de rayonnement civilisationnel, culturel et scientifique.

En 1798 les Français découvrent un pays pratiquement en voie de disparition et qui, rongé par les maladies et l'incurie de ses maîtres-esclaves, se vide lentement mais sûrement de sa population.

\section{Quels déséquilibres pour quel équilibre?}

Chaque type de société s'organise autour d'un point d'équilibre qui lui est propre et qui finit par déterminer simultanément son évolution interne et sa relation aux autres sociétés. La lutte pour le maintien de cet équilibre peut tout aussi bien être une source de développement ou de dépérissement. Dans la mesure où l'ensemble du système se réorganise constamment pour tendre vers l'équilibre, certaines modifications, parfois infimes, de sa structure peuvent modifier radicalement son orientation générale. 
L'équilibre dynamique de l'Égypte de l'époque comme d'ailleurs encore celui de l'Égypte actuelle s'appuie sur trois composantes :

1. Pour qui connaît l'islam dans son extrême diversité, l'Égypte est probablement le plus musulman des pays musulmans et certainement le plus musulman des pays arabes. Non pas pour des raisons institutionnelles ou théologiques ou par rapport à une quelconque mesure savante de l'orthodoxie, mais parce que de tout temps l'Égypte a été le berceau d'une conception religieuse de l'ordre de l'univers fortement marquée d'une part par le monothéisme, d'autre part par une forme de religiosité populaire qui a constitué la colonne vertébrale de sa résistance identitaire aux deux étrangers, celui qui vient du dehors et celui qui, né en elle, l'opprime. Les Égyptiens, quelles que soient leur confession et leurs convictions comptent probablement parmi les derniers peuples du monde à voir encore des apparitions de la Vierge Marie. Même athée, le rapport au monde d'un Égyptien est fondamentalement médiatisé par sa relation à Dieu. Dans Sabah el ward, Naguib Mahfouz met en scène un personnage, très vraisemblablement lui-même, peu pratiquant mais dont un autre personnage dit qu'il a, malgré son apparente indifférence en matière de religion, des «tripes musulmanes».

Cette religiosité diffuse et cette confiance absolue dans la médiation divine produit le pire lorsque le musulman passe du tawakkol 3la-llahau tawâkol,c'est-à-dire de la confiance qui pousse à agir du fait qu'on se sent en droit, ayant fait le maximum, de s'en remettre à Dieu sans cesser pour autant d'être responsable de ses actes à la résignation fataliste qui justifie l'inaction et dégage indûment la responsabilité. Ce que Bonaparte montre aux Égyptiens, c'est d'une part qu'on peut être un militaire efficace sans cesser pour autant d'être intelligent, d'autre part que la maîtrise du monde se situe à un niveau qui n'a rien à envier au niveau des représentations religieuses, et qu'il correspond parfaitement à l'esprit comme à la lettre du Coran. Il leur montre au jour le jour pourquoi l'équilibre où ils se trouvent est négatif et comment ils doivent faire pour revenir à la positivité. C'est ce message, qui pendant un peu plus d'un siècle, s'identifiera à la référence française des élites égyptiennes.

2. L'Égypte est un pays quasiment plat, relativement étendu - le double de la France militairement indéfendable mais suffisamment peuplé, structuré et homogène sur sa bande centrale habitable et exploitable - un trentième du territoire - pour absorber et digérer à terme les incursions étrangères qui ont souvent beaucoup de difficulté, une fois entrées, à repartir... L'Égypte utile est paradoxalement, avant le développement du transport aérien, aussi isolée qu'une île perdue dans l'Océan. La référence française, sous Mohammad Ali notamment, c'est aussi une option pour répondre à la question récurrente : comment se défendre?

3. L'Égypte n'a rayonné lorsqu'elle en a eu les moyens qu'en attirant à elle. Isolée mais sur une voie stratégique, l'une des branches de l'ancienne route de la soie et réservoir de richesses dans un environnement relativement stérile, l'exercice de son influence a été un cycle d'attraction, de transformation et de restitution. Cette fonction était au cœur du projet napoléonien et correspondait parfaitement à la vocation de l'Égypte. C'est celle que la France contemporaine essaie, après une longue absence, de ranimer mais avec une compétence et une énergie qui auraient fait sourire les savants et les soldats de Bonaparte...

\section{Le pari de Mohammad Ali}

Le fondateur indiscutable de l'Égypte moderne, malgré sa basse extraction, ses origines étrangères, sa mauvaise maîtrise de la langue arabe, son mépris de la vie humaine et des droits des gens ainsi que son illettrisme, est aussi le premier homme de pouvoir en Égypte à avoir tiré des leçons utiles de l'expédition de Bonaparte, à avoir jeté les bases d'un renouvellement de ses élites autochtones et à leur avoir rendu symboliquement 
l'initiative dans la reconstruction de l'Égypte et ce, à travers la France et avec des Français. Pressé par Edme-François Jomard, commissaire impérial pour la Description de l'Égypte, dès 1811, pour "envoyer une colonie en France et l'y laisser assez longtemps pour y puiser, malgré la différence des mœurs, une instruction complète »", il finit par accepter... en 1826. Les 44 premiers boursiers égyptiens resteront à Paris jusqu'en 1835 et constitueront le noyau de cette élite francophone et musulmane à travers laquelle la référence française s'est enracinée au plus profond de la sensibilité des classes moyennes et supérieures de l'Égypte moderne.

Mohammad Ali a débarrassé l'Égypte d'au moins quatre de ses plaies : l'ensemble de la fausse élite de son époque, la caste militaire des Mamelouks qu'il a fait massacrer en une soirée, la chape tétanisante et stérilisante de la domination ottomane - il a failli occuper Istanbul -, l'influence symbolique d'une Arabie d'opérette totalement étrangère à l'islam des origines - il a occupé La Mecque -; enfin il a rendu aux Égyptiens leur indispensable confiance dans leur pouvoir de nuire à qui leur ferait ombrage. Il a bâti, grâce aux Français, des usines, des voies de communication et une armée qui sera la première, depuis le califat fatimide, à déployer efficacement sur un théâtre d'opérations des soldats égyptiens armés d'armes égyptiennes et dirigés par un Égyptien, son fils Ibrahim.

\section{Le canal de Suez}

19 La présence française la plus conséquente et la plus longue en Égypte a été celle des Français de Suez, mais elle s'est développée sur le mode habituel de la présence coloniale européenne en Afrique et en Asie. Les Français du canal ont vécu pendant près d'un siècle comme si les Égyptiens, et notamment les musulmans égyptiens, n'existaient pas, d'où leur surprise lors de la nationalisation du canal par Nasser !

Malgré cela le canal a été longtemps une vitrine positive de la référence française. Ismaïlya, la ville des Français du canal, a été longtemps et tend à redevenir, après avoir été ravagée par les alliés israéliens de la France, l'une des plus belles, des plus propres et des mieux urbanisées des villes égyptiennes. Une oasis de civilisation à la française dans un pays devenu progressivement étranger aux civilisations qu'il avait pourtant portées à un rare degré d'épanouissement.

\section{Un Opéra pour Eugénie, des barrages sur le Nil, un Parlement, des chemins de fer, des postes : l'âge d'or du rayonnement français}

21 La France et les Français occuperont la première place dans la vie administrative, juridique, économique, culturelle et intellectuelle de l'Égypte pendant très exactement 25 ans sous les règnes de Sa3îd Pacha (1854-1863), fils de Mohammad Ali et Isma3îl Pacha (1863-1879), fils d'Ibrahim Pacha. C'est l'époque de la construction du canal, mais c'est aussi une époque d'autres grands travaux - régulation du Nil, création de voies de chemin de fer, d'un système postal moderne - et de bon nombre de réformes institutionnelles - abolition de l'esclavage, stabilisation de l'impôt agricole, création d'un Parlement - qui vont redonner à l'Égypte sa stature «naturelle» et lui faire retrouver la place qui lui revient. Les Italiens, les Autrichiens et les Anglais y participent, mais c'est la France et le français qui jouent les premiers rôles et occupent le devant de la scène. Et tout ce monde, y compris dans la haute administration 
égyptienne, ne parle que français. C'est aussi une époque de paix et d'indépendance - la tutelle ottomane n'est plus que symbolique. Lorsqu'Isma3îl Pacha inaugure l'Opéra du Caire dans la foulée de l'inauguration du canal de Suez en 1869, c'est aux côtés de l'impératrice Eugénie. Le rêve égyptien de Bonaparte qui se voyait empereur enturbanné d'Orient et d'Occident est relayé par le rêve égyptien d'Isma3îl attirant dans le berceau des civilisations toute la grandeur, la beauté et la modernité de l'Europe.

Mais l'intendance n'a pas suivi. La modernisation est encore plus chère que la guerre. L'Égypte s'y endettera jusqu'à y perdre son indépendance. Hier comme aujourd'hui, seules les très grandes puissances peuvent se permettre d'être déficitaires. Développée puis ruinée par les intérêts français, l'Égypte sera confisquée en 1882 par les Anglais.

On comprend mieux dans ce contexte que pour les élites égyptiennes en formation se mêleront longtemps, attachés à la France et au français, d'une part les rêves d'indépendance, de progrès, de beauté, de luxe, de plaisir et, par dessus tout de cette grandeur qu'ils n'ont jamais su assumer, d'autre part cette représentation de la culture, de la science, du savoir et du savoir-faire que la passion de Bonaparte et de ses savants pour l'Égypte a rendus pour ainsi dire indissociables du versant à la fois patrimonial et moderne de leur identité.

Le très pragmatique Milner le subodore, mais a du mal à l'admettre. Les Anglais aideront les Égyptiens à rendre leur système agricole aussi parfait que la nature le permet, ils leur permettront de triompher des principales maladies endémiques qui les ont décimés à travers les siècles - notamment la bilharziose -, respecteront scrupuleusement leurs deux religions et n'interféreront jamais dans leurs usages et coutumes. Ils n'en resteront pas moins, tout en étant souvent admirés et respectés, et jusqu'à leur départ en 1956, au mieux des Martiens, au pire des occupants haïs, profondément étrangers aux aspirations des Égyptiens, du peuple comme de l'élite.

\section{Une mosaïque d'immigrés francophones}

Le mouvement en faveur de la référence française ne concernait pas les seuls Égyptiens, coptes ou musulmans. Il concernait aussi cinq minorités qui joueront un rôle décisif dans le façonnement de la société égyptienne au $\mathrm{XIX}^{\mathrm{e}}$ siècle et dans la première moitié du XXe siècle : les juifs - notamment ceux qui étaient protégés par l'empire ottoman -, les Levantins qui ont trouvé refuge en Égypte à plusieurs reprises notamment dans le courant de la deuxième moitié du XIX $\mathrm{X}^{\mathrm{e}}$ siècle, les Grecs, très nombreux dans toute l'Égypte pendant toute la première moitié $\mathrm{du} \mathrm{XX}^{\mathrm{e}}$ siècle, les Italiens et les Arméniens. Pour diverses raisons ces cinq minorités adopteront le français comme langue véhiculaire commune, en grande partie sous l'impulsion des juifs et des écoles de l'Alliance israélite universelle. Ces minorités seront présentes à tous les échelons de la vie sociale. Dans le commerce, l'artisanat, les professions libérales mais aussi parmi les ouvriers et dans le mouvement syndical. Le dialecte égyptien tant dans ses versions cairote, alexandrine ou de Haute-Égypte a arabisé un nombre considérable de termes français utilisés par ces minorités dans différents domaines d'activité. L'arabe égyptien, l'un des éléments les plus importants de l'identité des Égyptiens et de leur rayonnement culturel dans le monde arabe pendant près d'un siècle porte de nombreuses traces du français référentiaire des élites comme du petit peuple d'Égypte ${ }^{5}$. 


\section{Un réseau exceptionnel d'écoles} pour qui l'Égypte - comme d'ailleurs pour bon nombre d'illettrés qui nous gouvernent ou dirigent nos entreprises - est un pays anglophone, sont souvent fort étonnés de la taille pour ne pas dire de l'existence du collège Saint-Marc, du collège de la SainteFamille, du collège de la Salle, du lycée français du Caire, du collège de la Mère de Dieu, du collège de Saint-Vincent de Paul, du collège des Frères, de l'école des Sœurs franciscaines, et j'en passe... Cours de récréation assez grandes pour deux matchs simultanés de football, salles de théâtre que leur envieraient bien des théâtres parisiens, couloirs interminables et salles de classes d'une capacité supérieure à 60 élèves. Étonnés également que plusieurs de ces collèges offrent un enseignement qui va de la maternelle au baccalauréat et de savoir qu'à leur création ils étaient mieux équipés que ne le sont aujourd'hui la plupart des lycées et collèges des banlieues de Paris, Lyon ou Marseille. Étonnés enfin de savoir que ces écoles n'étaient pas réservées aux chrétiens ou aux minorités étrangères, mais ont toujours accueilli une majorité écrasante d'Égyptiens et un très grand nombre de musulmans dont les parents ne parlaient pas un mot de français. Ce réseau, bien que souvent ignoré ou moqué par les représentants de la très laïque République, a construit un référentiel français en terre non francophone sans équivalent sur le globe terrestre.

\section{De 1919-1923 à 1952-1954 : des effendis aux militaires}

La première révolution de l'Égypte moderne, la révolution des effendis de 1919,voit descendre dans la rue des jeunes gens en tarbouche rouge et costume occidental pour la plupart instruits, parfois polyglottes, souvent francophones. Leur mouvement se transforme vite en un mouvement réellement populaire. C'est un moment exceptionnel, peut-être le seul de l'histoire de l'Égypte où le peuple se soit reconnu dans son élite et l'ait suivie. Il en sortira la fameuse délégation (al wafd) partie à Londres et à Paris réclamer l'indépendance de l'Égypte au nom de la déclaration en 14 points du 8 janvier 1918 de Thomas Woodrow Wilson, président des États-Unis, reconnaissant le droit des peuples à disposer d'eux-mêmes.

Le Wafd, même si bon nombre de ses grandes figures sont francophones ou anglophones, est d'abord l'espace où se constitueront autour d'une poignée d'intellectuels et d'universitaires les protocoles du discours public de l'arabe moderne pour l'Égypte d'abord mais aussi pour tout le reste de l'intelligentsia arabe. Un espace qui reléguera les langues étrangères, c'est-à-dire principalement le français à l'espace privé et aux territoires souvent complices dans le nouveau jeu politique du Palais et des minorités.

Parallèlement à l'évolution des effendis qui gardent intact ou se donnent un rapport positif à la langue élitaire tout en développant la langue nationale, un autre mouvement prend de l'ampleur au sein des élites. Alors que plusieurs réformateurs musulmans de la fin du XIX siècle et du début du XX ${ }^{\mathrm{e}}$ vont en France et écrivent sur la France dont ils parlent la langue, une cassure se produit une dizaine d'années après l'émergence du Wafd. Une partie non négligeable des élites musulmanes se sent menacée dans son identité retrouvée par l'européanisation accélérée de la société, par

Documents pour l'histoire du français langue étrangère ou seconde, 38/39 | 2007 
le fait que l'essor économique profite surtout aux minorités et que ce sont ces mêmes minorités qui s'instituent en médiateurs obligés entre l'Égypte et l'Europe, entraînant une marginalisation des musulmans dans plusieurs secteurs émergents de la vie publique. D'autre part, le contact avec les Européens met en jeu de nouveaux seuils de tolérance. Jusque dans les années 1920 les différences vestimentaires et le rapport à la sexualité entre occident et orient restent publiquement gérables et ne présentent de toute façon pas de disparités vraiment significatives. L'entre-deux-guerres creuse assez rapidement l'écart. L'Occident et l'Orient divergent dans leur conception même de la pudeur et de bon nombre de signes extérieurs par lesquels se manifeste le rapport à la dignité humaine. La frontière entre la sphère privée et la sphère publique connaît un déplacement significatif avec pour corollaire un rapport de forces permanent sur la place publique autour des droits et des limites de la libre disposition par chacun de son corps.

Problème radicalement nouveau. Il suffit pour s'en convaincre de relire la réponse, écrite là encore dans un français d'écrivain français, de Kassem Amine, champion toutes catégories de l'émancipation de la femme musulmane, à l'aube du XXe siècle, aux critiques de la société musulmane formulées par le duc d'Harcourt. La même critique formulée trente ans plus tard aurait choisi des angles d'attaque très différents - les mêmes que ceux qui peuplent aujourd'hui la production islamophobe - et la réponse, qui aurait eu du mal à trouver une plume musulmane aussi délicieusement francophone que celle de Kassem Amine, aurait pris les allures d'une guerre totale au mode de vie occidental.

31 Si les musulmans laïs et modernistes continuent à s'adresser à l'Occident - ils se sont même donné pour objectif d'en faire partie - et qu'ils le font toujours dans les langues qui ont marqué leur formation en tant qu'élites, les autres ont le plus souvent cessé de s'adresser à l'Occident athée et ne font plus du français, de l'anglais ou d'autres langues européennes qu'un usage strictement fonctionnel.

Cette évolution, entamée dans les années trente, va se poursuivre jusqu'à nos jours et priver progressivement le français de la grande majorité des musulmans égyptiens de sa valeur référentiaire en le ramenant dans le giron des minorités et des nostalgiques d'une époque définitivement révolue. Bref, disqualifier son aptitude à provoquer ou accompagner une nouvelle ouverture de la société musulmane aux savoirs et savoirfaire occidentaux.

\section{6 : la fin des minorités et l'ouverture à l'Amérique}

L'ère nassérienne (1954-1970) maintient le statut des langues hérité de la monarchie malgré son hostilité déclarée aux "agresseurs" franco-britanniques alliés et protecteurs d'Israël. Si les programmes des écoles françaises sont égyptianisés et que l'enseignement de la langue arabe, de la religion musulmane ou chrétienne et de l'histoire et de la géographie du pays y sont rendus obligatoires, la part de l'enseignement des langues étrangères ainsi que l'enseignement des disciplines scientifiques dans ces langues sont maintenus voire renforcés. Globalement l'enseignement est bien mieux équilibré que sous la monarchie - souvent, les élèves des écoles françaises parlaient mal l'arabe, ne savaient pas l'écrire, et ne connaissaient pas leur pays - et bien meilleur que ce qu'il deviendra à l'ère ultra-libérale actuelle produisant des élèves incapables de suivre des études supérieures en français -. 
34 La politique éducative nationaliste de Nasser a également pour effet de freiner considérablement le mouvement amorcé dans les années trente par les élites musulmanes pratiquantes et de gagner bon nombre de ses membres à une sorte de laïcité du troisième type qui constitue un compromis supportable entre le mode de vie athée et le mode de vie des croyants.

Enfin, cette formule assure une transition en douceur au sein des francophones euxmêmes parmi lesquels on va compter de moins en moins de minorités et de plus en plus de coptes et de musulmans sans que pour autant l'usage et la fonction du français ne régressent de manière significative. Pour ma promotion, en 1968, de la section de français de la faculté des Lettres de l'université du Caire, nous sommes, sur les cinq premiers, quatre musulmans et un copte. Et sur les quatre musulmans un seul représentant de l'ancien régime, c'est-à-dire de ceux qui n'ont pas été formés par Nasser mais plutôt contre lui, et un seul descendant de minorité immigrée.

Mais cet équilibre sera rompu brutalement par la course effrénée à l'enrichissement tous azimuts encouragée par le président Sadate à l'issue de la conclusion de la paix avec Israël. En quelques années les élites culturelles ont le choix entre l'émigration et la mendicité, une bonne moitié des classes moyennes se prolétarise, le tissu associatif tombe en lambeaux, des écoles privées aux tarifs rédhibitoires poussent comme des champignons et ne forment plus une élite du savoir et du savoir-faire mais des ghettos sociaux de dégénérés incultes. Parallèlement le réseau des écoles françaises et bon nombre d'anciennes écoles anglaises se désagrègent, ne parvenant plus à cibler leur public, à recruter, payer et former leurs enseignants. Certaines de ces écoles, jadis prestigieuses, n'enseignent même plus dans la langue qu'elles annoncent ou l'enseignent si mal qu'on a du mal à la reconnaître.

Parallèlement, les classes montantes ne sont plus tournées vers la référence française, même quand certains de leurs membres ont été formés dans les anciennes écoles françaises. N'ayant pas su profiter des années « Enrichissez-vous! » pour implanter une université française, les agents francophones en Égypte et les Égyptiens francophones se voient contraints d'envoyer les meilleurs élèves des écoles françaises qui en ont les moyens à... l'université américaine du Caire où ils supplanteront souvent par leur qualité les étudiants venus d'écoles anglaises... mais il ne sera plus question pour eux de miser sur ce mauvais cheval qu'est devenue la référence française.

Chrétien, musulman, athée, riche, pauvre, provincial, citadin, laïc, laïcard, islamiste, intégriste, radical, modéré, génie ou demeuré, la référence devient anglo-américaine. Ce que 74 années d'occupation britannique n'ont pas réussi à obtenir à cause du ferment de Bonaparte, quelques années d'incurie de politiciens français dont on ne risque pas de se souvenir un jour l'ont offert sur un plateau d'argent à une référence anglo-américaine qui anéantit la région beaucoup plus efficacement et sûrement que n'ont pu le faire quatre siècles de colonisation.

\section{Le français référentiaire de quelques familles musulmanes non francophones de bonne bourgeoisie}

Six composantes sont vraisemblablement nécessaires, quand on n'est pas francophone, pour parfaire son intégration dans l'univers du français et l'ériger en référence :

1) Le passage par une école française. 
2) La poursuite de ses études supérieures en français.

3) Avoir des parents ou des proches qui en tiennent lieu qui sont partiellement ou entièrement francophones.

4) Appartenir à un milieu où on peut se faire des amis francophones.

5) Avoir un usage professionnel du français.

6) Avoir effectué des séjours prolongés dans un pays francophone.

On peut certes avoir une pratique quasi parfaite du français sans satisfaire nécessairement à ces six conditions. On peut même comprendre parfaitement le français et en faire un usage tout à fait honorable en ne satisfaisant que deux de ces conditions.

41 Le plus remarquable dans l'Égypte de la période qui nous intéresse est le fait qu'il est possible de retrouver dans un nombre considérable de familles musulmanes ou coptes tout ou partie de ces cas de figure et avec la quasi totalité des configurations possibles et que c'est cela et non la situation relativement homogène du français des minorités francophones qui a fait du français une langue référentiaire pour une part non négligeable de la société égyptienne. C'est bien plus le fait que des familles qui ne parlent pas le français envoient leurs enfants dans une école française ou décident au sortir d'une école publique arabe que leurs enfants feront des études supérieures en français ou encore qu'un de leurs membres choisisse, n'ayant jamais fait d'études en français, de se donner les moyens de fréquenter un milieu francophone, de travailler en français ou de séjourner dans un pays francophone autrement que pour des raisons strictement économiques, qui construit une représentation élitaire attachée à la pratique du français.

42 C'est ce diplomate d'abord nommé à Londres, ayant pour première langue étrangère l'anglais et pour passion culturelle exclusive de toute autre l'Italie, mais formé par un ambassadeur polyglotte proche du Palais dont il donnera le nom à son fils, qui décide au terme de deux ans de séjour à Paris, que son fils apprendra le français avant même d'apprendre l'arabe.

43 C'est la petite-nièce du secrétaire général du Wafd, plusieurs fois Premier ministre, Nahas Pacha, qui se retrouve, alors que son père ne parle aucune langue étrangère et ne manque pas, en bon musulman, une seule de ses cinq prières quotidiennes, au collège de la Mère de Dieu.

44 C'est, parmi les descendants de croisements entre la famille de l'écrivain Mohammad 'Al Mouwilhi (Hadith 3issa Ibn Hicham - 1911) et des familles dont les titres de noblesse remontent aux 'achrâfs (Chérifs) de La Mecque, tous ces enfants, fils de magistrats, d'exploitants agricoles ou de petits rentiers, placés, de 1930 à 1960, dans des écoles françaises.

45 Enfin, c'est l'actuel gouverneur de la Banque centrale d'Égypte, qui est un ancien élève des jésuites. Mais celui-là a fait ses études supérieures à Harvard après avoir fait ses classes chez un autre ancien élève des jésuites, à la tête d'une banque privée et, pendant longtemps, de la chambre de commerce égypto... américaine.

46 Aucun des deux derniers n'a placé ses enfants dans des écoles ou des universités de langue française. Aucun des deux n'a de voiture française et aucun des deux ne passe plus ses vacances en France. La nouvelle élite socio-économique, même quand elle doit sa formation aux institutions issues du génie français des lendemains de la Révolution, n’a plus dans son horizon, de référence française. 


\section{BIBLIOGRAPHIE}

AL-JABARTI, 'Abd-al-Rahman (1979), Journal d'un notable du Caire durant l'expédition française (1798-1801) [Traduit et annoté par Joseph Cuoq - Préface de Jean Tulard], Paris : Albin Michel, $430 \mathrm{p}$.

DENON, Vivant et EL-GABARTI, Abdel Rahman (1998), Sur l'expédition de Bonaparte en Égypte Témoignages croisés et commentés par Mahmoud Hussein, Paris : Babel / Actes Sud, 335 p.

HARCOURT, duc d' (1893), L'Égypte et les Égyptiens,Paris : Plon, 305 p.

KAMEL PACHA, Moustafa (1906), Égyptiens et Anglais - Discours choisis de Moustafa Kamel Pacha,Paris : Librairie académique Didier - Perrin \& $C^{\text {ie }}, 330$ p.

MILNER, Sir Alfred [1892], L'Angleterre en Égypte - [Nous avons utilisé la traduction par M. F. Mazuc de la $5^{\mathrm{e}}$ édition, 1898, Paris : Plon, Nourrit \& $C^{\text {ie }}$ - Toulouse : Édouard Privat, 509 p.]

MINNAERT, E. (s.d. - \pm 1890 ), Le Caire et la justice internationale en Égypte, Bruxelles : P. Weissenbbuch, Imprimeur du Roi, $457 \mathrm{p}$.

Muséum National d'Histoire Naturelle (1998), Les savants en Égypte,Paris : Nathan, 144 p.

RIVAROL, Antoine de [1784] et (1995), Discours sur l'universalité de la langue française,in Académie de Berlin, De l'universalité européenne de la langue française, Corpus des œuvres de philosophie en langue française, Paris : Fayard, 127-186.

SOLÉ, Robert (1998), Les savants de Bonaparte,Paris : Seuil, 256 p.

VAUGELAS, Claude Favre de [1647], Remarques sur la langue française utiles à ceux qui veulent bien parler et bien écrire,Paris : Augustin Courbé et Vve Camusat - [Nous avons utilisé la réédition de 1981, Paris : Éditions Champ Libre, 363 p.]

\section{NOTES}

1. $1906: 21-43$.

2. Al Liwa / L'Étendard.

3. Al Hizb al Watani / Le Parti national.

4. Muséum, 1998 : 110.

5. Pour un exemple contemporain, il suffit de visionner dans sa version originale le film L'immeuble Yacoubian.

\section{RÉSUMÉS}

Depuis l'expédition de Bonaparte en Égypte et jusqu'à « l'ouverture » décrétée par le Président égyptien Anouar al-Sadate, soit pendant près de deux siècles, la langue française est considérée par les élites égyptiennes, toutes tendances et appartenances confondues, comme étant un 
vecteur exemplaire d'excellence. Le français et ce qu'il représente restera longtemps une référence malgré l'occupation britannique du fait qu'il aura été adopté par les lettrés musulmans, participera de la dynamique nationale du pays et sera la langue véhiculaire de toutes les minorités actives dans toutes les couches sociales. L'article analyse les ingrédients authentiquement égyptiens de la dynamique qui a donné au français son statut référentiaire ainsi que les raisons qui ont amené les élites égyptiennes à s'en détourner dans les années 1970.

Since Bonaparte's expedition in Egypt and until the " openness " policy of Egyptian President Anwar al-Sadate, that is for almost two centuries, French was considered by Egyptian élite, whatever could have been their tendencies or beliefs, as an exemplary vector of excellence. French and what it conveys will remain for a long time a reference in spite of British occupation because it will be adopted by well-read muslims, will take part in national dynamics of the country and become the lingua franca of all active minorities in all social strata. The paper analyses the genuine Egyptian ingredients of the dynamics that gave French its referential status as well as the causes that brought Egyptian élites to abandon it during the 70's.

\section{INDEX}

Mots-clés : langue française, statuts, élites égyptiennes, histoire, occupation britannique

Keywords : French language, status, Egyptian elites, history, British occupation

\section{AUTEUR}

\section{AMR HELMY IBRAHIM}

Université de Franche-Comté

\& Université Paris-Sorbonne

(Laboratoire LaLIC), France 